Nouveau:

TriXera

\section{Bain émollient}

Soin complémentaire de TriXera Crème pour les peaux sèches et atopiques

Les Laboratoires dermatologiques Avène proposent un nouveau soin pour les peaux sèches et atopiques: TriXera Bain émollient formulé pour apaiser, surgraisser et protéger l'épiderme.

Lancé en 2000, TriXera Crème émolliente est un soin destiné au traitement de la xérose dans la dermatite atopique du nourrisson, de l'enfant et de l'adulte.

L'objectif du produit est de restaurer la fonction barrière de l'épiderme pour atténuer la xérose et éviter l'apparition de nouvelles poussées de dermatite atopique.

Afin de proposer un traitement complet pour une prise en charge globale de la xérose atopique, les Laboratoires dermatologiques Avène lancent TriXera Bain émollient qui permet de commencer la thérapie dès le bain.

TriXera Bain émollient possède les mêmes ingrédients actifs que la crème pour offrir une triple action sur la xérose:

1) action anti-irritante: l'eau thermale Avène, grâce à ses propriétés anti-irritantes et apaisantes, atténue rapidement les irritations et les sensations de tiraillement;

2) action anti-prurigineuse: le glycocolle calme les sensations d'inconfort liées aux démangeaisons;

3) reconstruction de la barrière épidermique: le trio lipidique végétal, association de lipides végétaux purifiés, correspond aux éléments défaillants de la structure épidermique: des céramides, dont les chaînes les plus longues viennent s'intégrer dans les structures lamellaires pour les reconstituer de façon biomimétique; des acides gras essentiels, dont l'acide $\gamma$ linoléique qui s'intègre au niveau membranaire; du cholestérol végétal nécessaire à la bonne structure des lamelles intercornéocytaires et au «natural moisturizing factor».

De plus, l'apport d'huile végétale (huile de coco) renforce l'action surgraissante et émolliente.

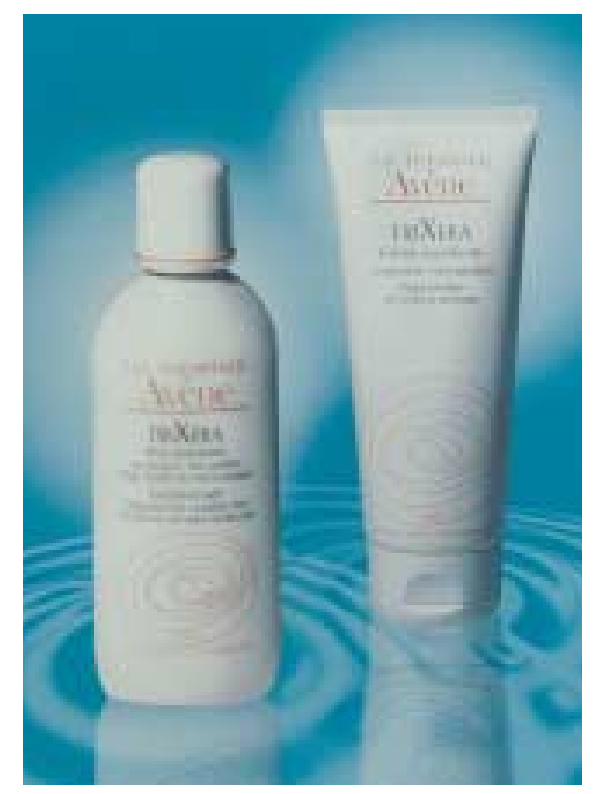

Pour tout complément d'information n'hésitez pas à nous contacter:

Pierre Fabre (Suisse) SA

21, route Sous-Riette

CH-1023 Crissier (Suisse)

Tél. 0216370202

Fax 0216370212

\section{Neu:

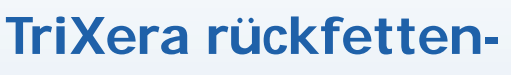 der Badezusatz}

\section{Zusatzpflege zu TriXera Creme für trockene und atopische Haut}

Die Laboratoires dermatologiques Avène haben ein neues Pflegeprodukt für trockene und atopische Haut entwickelt: TriXera rückfettender Badezusatz zur Beruhigung und zum Schutz der Haut.

Die TriXera weichmachende Creme wurde im Jahr 2000 lanciert und ist speziell zur Behandlung der Xerose bei Neurodermitis von Säuglingen, Kindern und Erwachsenen angezeigt.

Ziel des Produktes ist es, die Barrierefunktion der Epidermis wiederherzustellen, um die Xerose abklingen zu lassen und um das Auftreten von erneuten Schüben von Neurodermitis zu vermeiden.
Für die umfassende Behandlung der atopischen Xerose bieten die Laboratoires dermatologiques Avène den TriXera rückfettenden Badezusatz, mit welchem die Therapie gleich im Bad begonnen werden kann.

TriXera rückfettender Badezusatz besitzt die gleichen Aktivstoffe wie die Creme und hat daher eine Dreifachwirkung auf die Xerose:

1. Reizlindernde Wirkung: AvèneThermalwasser mildert dank seiner reizlindernden und hautberuhigenden Eigenschaften rasch Hautreizungen und das Gefühl von Hautspannen.

2. Juckreizstillende Wirkung: Das Glykokoll reduziert das durch den Juckreiz hervorgerufene Hautunbehagen.

3. Wiederaufbau der Hautbarriere: Das pflanzliche Lipidtrio, eine Kombination aus reinen pflanzlichen Lipiden, entspricht den der Hautstruktur fehlenden Elementen: langkettige Zeramide imitieren die Lamellenstruktur der Haut und stellen sie wieder her, indem sie sich in sie einfügen; essentielle Fettsäuren, davon die Linolsäure, fügen sich in die Membran ein; pflanzliches Cholesterin ist für die gute Struktur der interkorneozytären Lamellen und des «natural moisturizing factor» notwendig.

Die Zufuhr von pflanzlichem Öl (Kokosöl) verstärkt die rückfettende und weichmachende Wirkung.

Für weitere Auskünfte wenden Sie sich bitte an:

Pierre Fabre (Suisse) SA

21 , route Sous-Riette

CH-1023 Crissier (Schweiz)

Tel. 0216370202

Fax 0216370212 
Nouvelles de l'industrie

\section{Neues aus der Industrie}

\section{Propecia $^{\circledR}$ stabilise la chute des cheveux héréditaire de I'homme}

\section{Des résultats à long terme le confirment: la perte des cheveux n'est pas une fatalité!}

Des résultats après un traitement de 5 ans ont montré que la prise de $1 \mathrm{mg}$ de finastéride (Propecia ${ }^{\circledR}$ ) une seule fois par jour parvenait à stopper la progression naturelle de la perte des cheveux héréditaire chez l'homme. Cette étude, présentée au congrès annuel de l'European Academy of Dermatology and Venereology, le 11 octobre 2000 à Genève, est la plus longue étude randomisée et contrôlée contre placébo jamais effectuée à ce jour. Elle a pu démontrer que la perte des cheveux est stoppée chez 9 patients sous finastéride sur 10, et que chez 7 sur 10 les cheveux ont même repoussé. Les hommes sous placébo par contre ont dû se résigner à voir leurs cheveux continuer à disparaître.

«Propecia (finastéride, $1 \mathrm{mg}$ ) est le premier comprimé contre la perte des cheveux ayant un mode d'action causal», comme l'a bien précisé le Dr Ralph Trüeb, PD, Zurich, directeur du groupe de travail Trichologie des dermatologues suisses et président du symposium «Androgenetic Alopecia in Men: New Options in a New Millennium» à Genève. Pour lui, ce principe actif est donc «le prototype d'un traitement spécifique et rationnel, dont l'efficacité est prouvée dans la stabilisation de l'alopécie androgénétique». En inhibant spécifiquement la $5 \alpha$-réductase, le finastéride bloque la synthèse de dihydrotestostérone. Cela empêche la miniaturisation des follicules pileux touchés et stimule les follicules miniaturisés à produire des cheveux dits terminaux.
Dans l'étude présentée, qui s'est étendue sur 5 ans, 4 différentes méthodes d'évaluation ont été utilisées pour le comptage des cheveux. Le comptage assisté par ordinateur et l'examen des photographies par une commission d'experts dermatologiques ont ainsi donné des résultats reproductibles, ce qui est actuellement considéré comme standard international, selon le Prof. Jerry Shapiro, Vancouver, B.C., Canada. A cela sont venues s'ajouter l'impression clinique des médecins traitants et l'appréciation des patients eux-mêmes.

Après 5 ans, l'examen des photographies a révélé une stabilisation de la perte des cheveux chez $90 \%$ des hommes traités par Propecia. Le comptage des cheveux a montré une repousse capillaire chez $65 \%$ de ces hommes. Dans le groupe placébo par contre, la perte des cheveux a poursuivi son cours chez pratiquement tous les hommes.

Ce qui a été particulièrement frappant avec le temps, c'est la différence toujours plus marquée entre les groupes Propecia et placébo. Après 5 ans, la repousse des cheveux a atteint $31 \%$ en moyenne sous finastéride par rapport au placébo (ce qui correspond à 277 cheveux dans une zone alopécique représentative).

\section{«Une bonne impression»}

Pour les personnes traitées l'important n'est pas seulement le nombre absolu de cheveux, mais bien aussi l'impression personnelle. 213 hommes traités ont été satisfaits de leur status capillaire (frontal et occipital). 90\% des hommes traités ont constaté eux-mêmes un stop de la chute de leurs cheveux, et $75 \%$ même une repousse capillaire.

Le Dr Dowling B. Stough, Hot Springs, Ark., Etats-Units, a cité à ce propos un homme traité par finastéride à sa clinique dermatologique: «Même si on ne voit encore rien, mes cheveux semblent meilleurs et plus forts, et cela me donne une bonne impression.» Cette impression n'est pas un leurre, car la masse et le poids des cheveux augmentent de nouveau sous ce traitement.

L'incidence et la gravité des effets indésirables ont été identiques sous finastéride et sous placébo, comme l'a bien montré Shapiro. Ce qui corrobore les expériences positives déjà obtenues chez quelque 1,5 millions de patients traités par Propecia (finastéride) dans le monde entier.

\section{Une impression qui marque}

Le moment où l'homme constate pour la première fois que ses cheveux disparaissent à plus vive allure est un événement qu'il n'est pas prêt d'oublier, comme l'a affirmé le Prof. Jan Passchier, Rotterdam, Pays-Bas. Et pour les plus jeunes précisément, c'est le début d'une phase difficile pour leur image d'euxmêmes. L'intégrité capillaire joue un rôle important dans la communication non verbale. «La première impression marque souvent la vie privée et professionnelle», comme l'a expliqué ce psychiatre.

\section{Le plus tôt est le mieux}

Plus un traitement est mis en route précocement, plus les hommes profitent de la prévention de la poursuite de la perte de leurs cheveux, comme l'a bien dit le Prof. David Whiting, Dallas, Tex., EtatsUnis.

En effet, plus les follicules meurent, moins le traitement permettra de sauver de cheveux. Les résultats d'études le confirment également. Chez les patients traités d'abord par placébo pendant 1 an, le nombre de cheveux a bien augmenté dans les mêmes proportions après passage au finastéride. Mais ils n'ont pas tout à fait atteint le même niveau que le groupe traité d'emblée par finastéride.

\section{Traiter les cheveux et les patients}

Le Prof. Fernando Stengel, Buenos Aires, Argentine, s'est engagé pour une approche sensible des hommes demandant de l'aide de la part de leurs médecins traitants. Il s'agit de limiter l'importante attente à des proportions raisonnables, de manière à prévenir déceptions et problèmes de compliance. L'important est d'établir une relation médecin-patient stable. Car il faut attendre entre 6 et 12 mois avant qu'une première efficacité clinique du traitement de finastéride devienne manifeste. Dans le traitement de l'alopécie androgénétique, il faut voir loin, d'après Stengel. Mais l'expérience a montré que cela ne fait aucun obstacle, pour autant que la préparation ait été adéquate. 


\section{Propecia $^{\circledR}$ stabilisiert den anlagebedingten Haarverlust des Mannes}

\section{Langzeitdaten signalisieren: Haarverlust ist kein Schicksal!}

Langzeitdaten über einen Behandlungszeitraum von 5 Jahren zeigten, dass durch die Einnahme von einmal täglich $1 \mathrm{mg}$ Finasterid (Propecia ${ }^{\circledR}$ ) die natürliche Progression des anlagebedingten Haarverlustes beim Mann aufgehalten werden konnte. Die auf der Jahresversammlung der European Academy of Dermatology and Venereology am 11. Oktober 2000 in Genf vorgestellte Untersuchung ist die bisher längste hierzu durchgeführte randomisierte plazebokontrollierte Studie. Darin konnte gezeigt werden, dass bei 9 von 10 Patienten unter Finasterid weiterer Haarverlust gestoppt wurde und bei 7 von 10 das Haarwachstum sogar wieder zunahm. Im Gegensatz dazu mussten fast alle mit Plazebo behandelten Männer einen weiteren Haarverlust hinnehmen.

«Propecia (Finasterid, $1 \mathrm{mg}$ ) ist die erste Tablette gegen Haarverlust mit einem kausalen Wirkansatz», machte PD Dr. Ralph Trüeb, Zürich, Leiter der Arbeitsgruppe Trichologie der Schweizer Dermatologen und Vorsitzender des Symposiums «Androgenetic Alopecia in Men: New Options in a New Millennium» in Genf deutlich. Für ihn ist dieser Wirkstoff daher «der Prototyp einer spezifischen und rationalen Therapie mit erwiesener Wirksamkeit bei der Stabilisierung des androgenetisch bedingten Haarverlustes». Finasterid verhindert über eine spezifische Hemmung der $5 \alpha$-Reduktase die Bildung von Dihydrotestosteron. Damit werden die Miniaturisierung betroffener Haarfollikel gestoppt und bereits miniaturisierte Follikel zur Bildung von sogenannten Terminalhaaren aktiviert.

In der vorgestellten 5-Jahres-Studie wurden zur Haarzählung vier verschiedene Evaluationsmethoden eingesetzt. So liefern die computergestützte Messung sowie die fotografische Beurteilung durch ein dermatologisches Expertenteam reproduzierbare Ergebnisse, wie sie heute als internationaler Standard gelten, so Prof. Jerry Shapiro, Vancouver, B.C., Kanada. Darüber hinaus wurde der klinische Eindruck durch die behandelnden Ärzte sowie die Männer selbst bewertet.

Nach 5 Jahren zeigte die fotografische Beurteilung bei 90\% mit Propecia behandelten Männern eine Stabilisierung des Haarverlusts. Die Haarzählung zeigte bei $65 \%$ der Männer ein erneutes Haarwachstum. Im Gegensatz dazu wurde in der Plazebogruppe bei fast allen Männern ein weiterer Haarverlust beobachtet.

Besonders einprägsam war der im Zeitverlauf immer grösser werdende Unterschied zwischen der mit Propecia behandelten Gruppe und der Plazebogruppe. So betrug nach 5 Jahren die Zunahme der Haarzahl unter Finasterid gegenüber Plazebo im Mittel 31\% (was 277 Haaren in einem repräsentativen Bereich mit Haarverlust entspricht).

\section{«Ein gutes Gefühl»}

Wichtig für die Behandelten ist nicht nur die absolute Zahl der Haare, sondern auch der persönliche Eindruck. 213 der behandelten Männer waren mit dem Haarstatus (Stirnansatz und Hinterkopf) und ihrem Aussehen zufrieden. 90\% der behandelten Männer bemerkten selber einen Stopp des weiteren Haarverlusts, $75 \%$ stellten gar weiteres Haarwuchstum fest.

Dr. Dowling B. Stough, Hot Springs, Ark., USA, zitierte hierzu einen Mann, der an seiner Hautklinik mit Finasterid behandelt wurde: «Auch wenn man noch nichts sieht, meine Haare fühlen sich besser und stärker an, und das gibt mir ein gutes Gefühl.» Dieser Eindruck trügt nicht, denn auch die Haarmasse und das Gewicht nehmen unter der Behandlung wieder zu.

Inzidenz und Schweregrad unerwünschter Wirkungen entsprachen derjenigen von Plazebo, hob Shapiro hervor. Dies entspricht den positiven Erfahrungen, wie sie bei den weltweit etwa 1,5 Millionen mit Propecia (Finasterid, $1 \mathrm{mg}$ ) behandelten Patienten gemacht wurden.

\section{Ein Eindruck, der prägt}

Der Augenblick, in dem ein Mann zum ersten Mal bemerkt, dass sein Haarausfall zunimmt, ist für die meisten ein Ereignis, das sie nicht so leicht vergessen, bestätigte Prof. Jan Passchier, Rotterdam, Niederlande. Gerade für die Jüngeren ist dies oft der Beginn einer Phase, in der das eigene Selbstverständnis auf eine harte Probe gestellt wird. Denn volles Haar spielt eine wichtige Rolle in der nonverbalen Kommunikation. «Der erste Eindruck prägt oft, privat und im Berufsleben», erklärte der Psychiater.

\section{Je eher, desto besser}

Je eher mit einer Behandlung begonnen wird, desto mehr profitieren die Männer auch im Sinne der Prävention eines weiteren Haarverlustes, hob Prof. David Whiting, Dallas, Tex., USA, hervor. Denn je mehr Follikel absterben, desto weniger Haare können mit der Behandlung erreicht werden. Dies bestätigen auch die Studienergebnisse. Bei denjenigen Patienten, die zunächst 1 Jahr lang mit Plazebo behandelt wurden, wurde zwar nach der Umstellung auf Finasterid eine ebenso rasche Zunahme der Anzahl der Haare festgestellt. Sie erreichten jedoch nicht mehr ganz das Niveau der Gruppe, die von Anfang an mit Finasterid behandelt wurde.

\section{Die Haare und den Patienten behandeln}

Für einen sensiblen Umgang mit den hilfesuchenden Männern durch die behandelnden Ärzte setzte sich Prof. Fernando Stengel, Buenos Aires, Argentinien, ein. Es komme darauf an, die grosse Erwartungshaltung auf ein vernünftiges Mass zu beschränken, um Enttäuschungen und Complianceprobleme zu vermeiden, und eine stabile Arzt-PatientenBeziehung aufzubauen. Denn es kann 6-12 Monate dauern, bis sich eine erste klinische Wirksamkeit unter einer Behandlung mit Finasterid zeigt. Bei der Behandlung der androgenetischen Alopezie muss man langfristig denken, so Stengel. Die Erfahrung hat jedoch gezeigt, dass dies bei entsprechender Vorbereitung kein Therapiehindernis darstellt. 


\section{Antiinflamma- torische Wirkung von Adapalen wichtig für die Behandlung der Acne papulo- pustulosa}

Ausschlaggebend für die Beurteilung von Aknetherapeutika ist ihre Wirkung auf die verschiedenen pathogenetischen Krankheitsursachen. Substanzen wie Adapalen (enthalten in Differin ${ }^{\circledR} \mathrm{Gel}$ und Creme), die an mehreren entscheidenden Punkten der Akneentstehung ansetzen, nehmen in der Behandlung leichter und mittelschwerer Akneformen daher einen hohen Stellenwert ein, so die Auffassung von Privatdozent Dr. Stephan Sollberg von der Hautklinik Schwerin. Adapalen, ein Naphtoesäureabkömmling, zeichnet sich durch die Inhibition der Proliferation, die Modulation der Differenzierung der Keratinozyten sowie eine gute komedolytische und antiinflammatorische Wirkung aus. Letztere spielt insbesondere in der Behandlung der Acne papulopustulosa und in Kombination mit einer systemischen Therapie der Acne conglobata eine entscheidende Rolle.
In der Behandlung haben Wirkstoffe wie Adapalen, die auf die wichtigen pathogenetischen Ursachen der Akne einwirken, einen besonderen Stellenwert. Drei für die Entstehung der Akne zentrale Mechanismen sollten durch diese Therapeutika in jedem Fall verhindert bzw. verbessert werden, so die Auffassung von Privatdozent Dr. Stephan Sollberg von der Hautklinik Schwerin.

1. Die androgenabhängige Volumenzunahme der Talgdrüsen und die damit einhergehende Steigerung der Lipidsynthese;

2. Die noch weitgehend ungeklärte Proliferationssteigerung besonders der infundibulären Keratinozyten, die zur Ausbildung eines epidermalen Verhornungstyps führt und durch den daraus resultierenden gestörten Abfluss der produzierten Lipide die Entwicklung von Komedonen fördert.

3. Die quantitative Zunahme der residenten Keimflora, insbesondere von Propionibacterium acnes, die Entzündungen des Komedos hervorrufen kann.

\section{Pathogenetische Ursachen sind schweregradabhängig zu therapieren}

Je nach Schweregrad der Akne werden diese wünschenswerten Wirkansätze in der Behandlung jedoch unterschiedlich gewichtet. «Während bei allen Formen der Akne die Talgdrüsenproduktion reduziert und die Proliferationssteige- rung gehemmt werden müssen, spielt die antiinflammatorische Wirkung eines Aknetherapeutikums vor allem bei der Acne papulopustulosa und der Acne conglobata - die jedoch vorwiegend systemisch behandelt wird - eine entscheidende Rolle», erläutert Sollberg.

\section{Gute antiinflammatorische Wirkung von Adapalen}

In-vitro-Studien weisen darauf hin, dass Adapalen eine bessere antiinflammatorische Wirkung zeigt als topisch zu applizierendes Tretinoin. Adapalen hemmt die Lipoxygenase und darüber hinaus auch die Wanderung polymorphkerniger Leukozyten. «Neben dieser guten antiinflammatorischen Wirkung zeichnet sich Adapalen ausserdem durch eine hohe chemische und photochemische Stabilität sowie ein relativ geringes Irritationsspektrum aus. Dies macht den Wirkstoff zu einem herausragenden topischen Therapeutikum zur Behandlung auch der mittelschweren Akne, und das bei einem Nebenwirkungsspektrum, das sehr viel günstiger ist als bei einem systemisch wirkenden Therapeutikum», so das Resümee von Sollberg.

Für die Schweiz wenden sie sich bitte an: Galderma SA (Suisse)

Postfach 492

CH-6330 Cham (Schweiz)

Tel. 0417803828

Fax 0417804189 